However, most postpartum and non-obstetric postsurgical infections occur after hospital discharge. Decreasing lengths of hospital stay may further compromise detection of these infections. Several methods for postdischarge surveillance of postpartum infections have been evaluated.

Yokoe et al. used the inpatient and outpatient data collected by a health maintenance organization (HMO) to identify postpartum infections and describe the epidemiology of these infections. Screening was done on automated ambulatory medical records, hospital and emergency room claims, and pharmacy records of 2,826 HMO members who gave birth during a 30-month period. Full-text ambulatory records were reviewed for the 30 -day postpartum period to confirm infection status for a weighted sample of cases.

The overall postpartum infection rate was $6.0 \%$, with rates of $7.4 \%$ following cesarean section and $5.5 \%$ following vaginal delivery. Rehospitalization; cesarean delivery; antistaphylococcal antibiotics; diagnosis codes for mastitis, endometritis, and wound infection; and ambulatory blood or wound cultures were important predictors of infection.

The researchers concluded that the use of automated information routinely collected by HMOs and insurers allows efficient identification of postpartum infections not detected by conventional surveillance.

FROM: Yokoe DS, Christiansen CL, Johnson R, et al. Epidemiology of and surveillance for postpartum infections. Emerg Infect Dis 2001;7:837-841.

\section{Nutrition and Nosocomial Infections in Critically Ill Patients}

Caparros et al. from Madrid, Spain, conducted a study to evaluate the effects of a high-protein formula enriched with arginine, fiber, and antioxidants compared with a standard high-protein formula in early enteral nutrition in critically ill patients. Two hundred twenty patients were enrolled in a prospective, multicenter, single-blind, randomized trial in 15 Spanish intensive care units (ICUs). The primary end points were the incidence density rates of nosocomial infections, ICU and hospital length of stay, ICU and in-hospital mortality, and mortality at the 6-month follow-up.

The patients in the control and study groups had similar baseline characteristics. The study group had a lower incidence of catheter-related sepsis $(0.4$ episodes/1,000 ICU-days) than did the control group (5.5 episodes/1,000 ICU-days), with a relative risk (RR) of 0.07 (95\% confidence interval $\left[\mathrm{CI}_{95}\right], 0.01$ to $0.54 ; P<$ $.001)$. There were no differences in the incidence of ventilator-associated pneumonia, surgical infection, bacteremia, or urinary tract infections between the two groups. ICU mortality (16\% in the study group vs $21 \%$ in the control group; $\mathrm{RR}, 1.5 ; \mathrm{CI}_{95}, 0.7$ to 2.9 ) and in-hospital mortality (21\% in the study group vs $30 \%$ in the control group; $\mathrm{RR}, 1.6 ; \mathrm{CI}_{95}, 0.9$ to 3 ) were similar without differ- ences in survival at the 6-month follow-up (75\% in the study group vs $68 \%$ in the control group, $P=.15$ ). Patients in the study group who were treated for 2 or more days showed a strong trend for better survival at the 6 -month follow-up ( $76 \%$ in the study group vs $67 \%$ in the control group, $P=.06$ ). Medical patients treated with the study diet had better survival than did medical patients in the control group (76\% in the study group vs $59 \%$ in the control group, $P<.05$ ).

The authors concluded that critically ill patients fed a high-protein diet enriched with arginine, fiber, and antioxidants had a significantly lower catheter-related sepsis rate than did patients fed a standard high-protein diet. There were no differences in mortality or ICU and hospital length of stay. The subgroup of patients fed the study diet for more than 2 days showed a trend toward decreased mortality.

FROM: Caparros T, Lopez J, Grau T. Early enteral nutrition in critically ill patients with a high-protein diet enriched with arginine, fiber, and antioxidants compared with a standard high-protein diet: the effect on nosocomial infections and outcome. Journal of Parenteral and Enteral Nutrition 2001;25:299-308.

\section{Determinants of $S$. aureus Nasal Carriage}

Cole et al. from the UCLA School of Medicine screened 230 donors of diverse ethnic and socioeconomic backgrounds and identified 62 (27\%) whose nasal secretions were colonized by Staphylococcus aureus. In 18 donors in whom the various regions of the nasal luminal surface were separately sampled, the predominant region of $S$. aureus colonization was the moist squamous epithelium on the septum adjacent to the nasal ostium. Nasal fluid from carriers was defective in killing endogenous $S$. aureus and nasal carrier isolates of $S$. aureus but not a laboratory $S$. aureus strain.

Transmission electron microscopy revealed that $S$. aureus isolates incubated in nasal fluid from carriers for 2 hours at $37^{\circ} \mathrm{C}$ were less damaged than those incubated in noncarrier fluid and were coated with an electron-dense layer. Compared with that from healthy donors and patients with acute rhinitis, nasal fluid from carriers contained elevated concentrations of the neutrophil-derived defensins human neutrophil peptides 1 to 3 (47-fold and 4fold increases, respectively), indicative of a neutrophilmediated inflammatory host response to $S$. aureus colonization. The concentration of the inducible epithelial antimicrobial peptide human beta-defensin 2 was also highly elevated compared with that in healthy donors, in whom the level was below the detection limit, or patients with acute rhinitis (6-fold increase). Thus, $S$. aureus takes hold in nasal fluid that is permissive for colonization and induces a local inflammatory response that fails to clear the colonizing bacteria.

FROM: Cole AM, Tahk S, Oren A, et al. Determinants of Staphylococcus aureus nasal carriage. Clin Diagn Lab Immunol 2001;8:1064-1069. 